\title{
IDENTIDAD PROFESIONAL EN ESTUDIANTES DE TRADUCCIÓN CHILENOS: POSICIONAMIENTO, PERCEPCIONES Y VALORACIONES INICIALES
}

\author{
Professional identity in Chilean translation trainees: initial positioning, \\ PERCEPTIONS AND VALUATIONS
}

IDENTITÉ PROFESSIONNELLE DES ÉTUDIANTS DE TRADUCTION CHILIENS: POSITIONNEMENT, PERCEPTIONS ET ÉVALUATIONS INITIALES

\section{Néstor Singer Contreras}

MA TESOL, The University of

Manchester, Reino Unido.

Profesor asociado, Departamento de Lingüística y Literatura, Universidad de Santiago de Chile, Chile. nestor.singer@usach.cl

https://orcid.

org/0000-0001-7772-9906

\author{
Vania López Cañete \\ Magíster en Psicología Clínica, \\ Universidad Adolfo Ibáñez, Chile. \\ Psicóloga y asistente de \\ Investigación, Departamento de \\ Lingüística y Literatura, Universidad \\ de Santiago de Chile, Chile. \\ vaniaalopezcanete@gmail.com \\ https://orcid. \\ org/0000-0001-6430-5792

\section{Rosa Basaure Cabero} \\ Doctora en Estudios Americanos, con \\ énfasis en Relaciones Internacionales, \\ Universidad de Santiago de Chile, \\ Chile. \\ Profesora asistente, Departamento \\ de Lingüística y Literatura, \\ Universidad de Santiago de Chile, \\ Chile. \\ rosita.basaure@usach.cl \\ https://orcid. \\ org/0000-0003-2501-9456
}

\begin{abstract}
RESUMEN
El campo del desarrollo afectivo en la formación de traductores es un tema poco explorado, en particular el desarrollo de la identidad profesional en dicho proceso de formación. En ese sentido, este artículo presenta una primera indagación fenomenológica en torno al desarrollo de la identidad profesional del traductor en formación. En concreto, el estudio examina las narrativas sobre sí mismos respecto a la autodescripción y sus percepciones acerca del traductor profesional y acerca de su formación hasta el momento de este estudio, vale decir, marzo de 2018. Para ello, se realizan entrevistas semiestructuradas a un grupo de 9 alumnos de tercer año de un pregrado en Traducción en Chile. Los resultados dan cuenta de que los estudiantes: 1) se describen como personas tímidas e inseguras, con dificultad para socializar; 2) presentan una visión idealizada de la figura del traductor profesional, que genera conflicto con sus autodescripciones; 3 ) experimentan una brecha entre el miedo a la alteridad y el deber agenciar para cumplir con sus demandas académicas y personales, y 4) valoran positivamente la formación recibida hasta la fecha. Se concluye que estos elementos constituyen un insumo valioso para la planificación de asignaturas de Traducción en las siguientes etapas del programa.
\end{abstract}

Palabras clave: identidad profesional; narrativas; formación de traductores; traducción en Chile.

\section{Abstract}

Affective development in translator education is an issue that has been under-researched, particularly the development of professional identity among translators in training. This article presents a phenomenological study on how translator trainees develop their professional identity. This study examines their narratives concerning their self-descriptions and perceptions on professional translator and their training at the start of this study -i.e. March 2018. To do this, semi structured interviews were conducted with a group of nine third-year translation trainees in an undergraduate Translation program in Chile. Results show that students (a) describe themselves as shy, insecure individuals, who find it difficult to socialise; (b) present 
Esta publicación se adscribe al Proyecto DICYT 031851SC (2018-2020) "Identidad del traductor: una comprensión fenomenológica del posicionamiento del estudiante hacia su egreso" de la Universidad de Santiago de Chile. an idealised vision of the professional translator, which is misaligned with their self-perceptions; (c) experience an emotional conflict between the fear to alterity and their agency to fulfil their academic and personal commitments; and (d) prize the training received so far. It is concluded that these elements make up for a valuable input to plan translation course units at later stages of the program.

Keywords: professional identity; perceptions; narratives; translator education; translation in Chile.

\section{RÉSUMÉ}

Le champ du développement affectif dans la formation de traducteurs est un domaine peu exploré, particulièrement le développement de l'identité professionnelle des traducteurs en formation. Le présent article présente une première enquête phénoménologique au sujet du développement de l'identité professionnelle des traducteurs en formation. Cette étude analyse ses narratives concernant leur auto-description, leurs perceptions sur le traducteur professionnel et leur formation jusqu'au début de cette étude, c'est-à-dire en mars 2018. À cet effet, des entretiens semi-structurés ont été effectués sur un groupe de neuf étudiants de 3ème année d'un programme d'études de traduction chilien. Les résultats indiquent que les étudiants 1) se décrivent eux-mêmes comme des personnes timides, qui manquent de confiance en eux et qui ont des difficultés pour sociabiliser, 2) ils présentent une vision idéalisée de la figure du traducteur professionnel qui génère des conflits avec comment ils se décrivent eux-mêmes, 3) ils éprouvent une tension émotionnel entre le peur de l'altérité et leur capacité de répondre aux exigences académiques et personnelles, et 4) ils accordent de l'importance à leur formation ils ont reçu jusqu'au date. Il est conclu que ces éléments constituent une contribution de valeur pour la planification des matières de traduction dans les dernières étapes du programme.

Mots clés : identité professionnelle ; perceptions ; narratives ; formation des traducteurs; traduction au Chili. 


\section{Introducción}

Como parte de los países miembros de la Comunidad de Estados Latinoamericanos y Caribeños (CELAC), Chile se posiciona como una plataforma de servicios a nivel global. Para esto resulta indispensable contar con el capital humano avanzado que permita al país participar en un mercado mundial. En este contexto, el rol del traductor como mediador lingüístico y cultural alcanza una relevancia importante. No obstante, hoy en Chile existe una carencia de lineamientos en relación con la formación de traductores profesionales, por cuanto los programas determinan arbitrariamente, desde la perspectiva de la experiencia de sus diseñadores curriculares, qué es lo más pertinente, según lo que ellos creen son las necesidades en el mercado e institucionales. Lo anterior supone una tensión entre dos elementos: por una parte, figuran los objetivos a nivel país, relacionados con la internacionalización, el fomento y el fortalecimiento del comercio internacional de servicios en las áreas principales de intercambio económico (Subsecretaría de Relaciones Económicas Internacionales, 2020); por otra, el cómo las instituciones que ofrecen programas de pregrado en Traducción forman sus profesionales según las últimas tendencias del mercado. Esto, a su vez, genera incertidumbre respecto al tipo de educación recibida en las instituciones de formación superior y, posteriormente, acerca de su desempeño al insertarse en el mundo profesional.

Los estudios realizados por Diéguez et al. (2014, 2015) establecen que la mayoría de los traductores profesionales chilenos trabaja de modo independiente, en lugar de emplearse tiempo completo en agencias de traducción. En ambos escenarios, se produce un campo laboral competitivo, donde resulta vital poder desempeñarse de manera efectiva e integral como traductor profesional. No obstante, existe una cantidad importante de traductores egresados que se dedica a llevar a cabo otro tipo de labores lingüísticas, o bien no ejerce la profesión de traducción, debido que, a su juicio, presentan una serie de debilidades en su competencia traductora.
Esto puede deberse a varios factores, como la falta de explicitación de los objetivos de los sectores públicos y privados del país en materias de traducción; la ausencia articulación de dichos objetivos con los programas de formación de traductores; la baja competencia en las lenguas con las que trabajan, o el impacto de los programas de Traducción en el desarrollo de su identidad profesional como traductores, o sea, cómo los estudiantes concluyen sus estudios sintiéndose o no traductores.

Hasta esta fecha, solo recientemente se ha empezado a incursionar en el estudio del impacto de la autoeficacia en la formación de los traductores. Este concepto, entendido como el grado de confianza percibido por los alumnos en cuanto a su desempeño en algún oficio, como, en este caso, el de traductores, ha sido discutido de manera incipiente en la literatura (Atkinson, 2012, 2014; Muñoz, 2014), pero no se había integrado ampliamente al currículum de la formación de traductores, debido a las complejidades que presenta su naturaleza subjetiva (Way, 2009). Es solo hasta el trabajo de Haro-Soler (2017a, 2017b, 2018) que la autoeficacia se analiza y estudia en forma sistemática como factor relevante en el proceso de desarrollo de formación del traductor. Sin embargo, su impacto en la identidad profesional del traductor no ha sido documentado y, hasta el momento de la redacción de este trabajo, no existe literatura que aborde la evidencia del modo en que los traductores configuran su identidad durante sus estudios de pregrado en Latinoamérica, en particular en Chile.

En este sentido, esta publicación se enmarca dentro de un estudio fenomenológico que persigue explorar el desarrollo de la identidad profesional en un grupo de alumnos de pregrado en Traducción en un contexto chileno durante los últimos tres años de sus programas y, posteriormente, identificar cómo esta imagen, construida durante sus estudios de pregrado, incide en su sentido de autoeficacia. En esta misma línea, el artículo tiene como objetivo presentar el resultado del análisis de las concepciones que los alumnos de tercer año del programa de Lingüística Aplicada a la Traducción, de la Universidad de Santiago 
de Chile, manifiestan en sus narrativas en relación con su identidad profesional. Esto corresponde a un primer acercamiento a la comprensión de sus percepciones de las experiencias vividas durante su formación como traductores, lo queservirá como referencia para estudiar la evolución de las narraciones en el resto del proceso formativo. En consecuencia, las preguntas que orientan esta primera fase de trabajo ${ }^{1}$ y este artículo son:

1. ¿Cómo se sienten y posicionan los alumnos de tercer año de un programa de Traducción chileno antes de iniciar su formación en traducción?

2. ¿Cómo conceptualizan la figura del traductor profesional y la comparan con su identidad como estudiantes del programa hasta su punto actual de formación?

3. ¿Qué valor le atribuyen a su formación y que expectativas les genera el enfrentarse a la práctica de la traducción?

Las respuestas a tales interrogantes perfilarían a este trabajo como el primer estudio multidisciplinario chileno que aborda, a la fecha, la cognición del traductor en su etapa formativa, con aportes de la lingüística aplicada, la teoría de la traducción, la psicología y la pedagogía.

\section{Marco teórico}

Esta sección comprende los conceptos teóricos que subyacen a este estudio. Primero, se contextualiza este trabajo dentro de la formación de traductores, en particular las nociones de autoeficacia e identidad profesional. En el segundo apartado

1 La primera fase de trabajo corresponde al anclaje de los participantes con relación a cómo conciben al traductor, sus experiencias a la fecha y el valor que le asignan a su formación. La segunda fase se ocupa de la aplicación de cuatro rondas de entrevistas a los participantes, una al final de cada semestre académico, para evaluar la evolución de sus percepciones a lo largo de su formación. La tercera etapa consiste en la aplicación de una entrevista de salida, con un enfoque holístico, en donde los alumnos realizan un proceso reflexivo de lo vivido durante su formación. se exploran los componentes fenomenológicos que se conjugan en las experiencias que configuran dicha identidad. La tercera parte describe cómo dicho desarrollo identitario se lleva a cabo en la adolescencia tardía, que corresponde al rango etario en que se realiza este trabajo. Luego se problematiza la agencia de los individuos en dicha etapa de desarrollo, así como su anclaje emocional durante el proceso.

\section{Competencia traductora y autoeficacia}

Se ha estudiado poco sobre la dimensión afectiva y la traducción, entendida como lo que sienten y piensan los estudiantes de Traducción, así como su posible incorporación en la formación de traductores. A pesar de que el concepto de competencia traductora (СТ) ha evolucionado en los últimos 40 años, existen pocos estudios en relación con el rol que esta dimensión afectiva podría tener en el desarrollo de aquella competencia. El grupo PACTE (Proceso de Adquisición de la Competencia Traductora y Evaluación) (2017) propone, en su modelo de CT, los denominados "componentes físico-psicológicos", entre los que figuran los "rasgos aptitudinales como la curiosidad intelectual, perseverancia, rigor, espíritu crítico y confianza en sí mismo, la habilidad para medir las capacidades propias, motivación, etc.” (p. 40; nuestra traducción). Este modelo es uno de los pocos en que se reconocen estas características, las que se relacionan con la configuración de la identidad profesional.

Pese a que se ha estudiado acuciosamente en educación y enfermería, la identidad profesional no ha sido investigada en la formación de traductores. Tan et al. (2016) definen la identidad profesional como "el sí mismo que se ha desarrollado con un compromiso a desempeñarse competentemente y legítimamente en el contexto de la profesión" (p. 1505; nuestra traducción), es decir, el quién ha sido, es y será una persona en su proceso formativo, y proponen un marco de cinco factores, que dan cuenta de elementos clave en dicho proceso (p. 1507): 1) conocimiento sobre las prácticas profesionales, 2) consideración del profesional como modelo, 3 ) experiencia en la profesión, 4) preferencia por la profesión, y 5) autoeficacia 
profesional. Esta última es particularmente crucial para la configuración de la identidad profesional, ya que se refiere a "la confianza en las capacidades propias para ejecutar acciones y producir resultados en una tarea deseada" (Wood et al., 2000, p. 431). En traducción, esto implicaría qué tanta confianza tienen los estudiantes en sus habilidades para traducir (Haro-Soler, 2017a), y desde allí se puede explorar parte de los modos en que conceptualizan la profesión misma.

Sin embargo, aunque la autoeficacia se ha abordado en la literatura relacionada con estudios en traducción (Atkinson, 2012, 2014; Haro-Soler, 2017a, 2017b, 2018; Muñoz, 2014), no se ha integrado en una problematización macro, que se perfila como uno de los temas más complejos en la formación de traductores: cómo los traductores terminan su formación inicial identificándose (o más bien, sintiéndose) traductores o no. Esto puede deberse al hecho de que la autoeficacia no puede considerarse un producto estático y finito de la formación, sino más bien un proceso constante, que se extiende más allá de las etapas iniciales de la misma.

En consecuencia, para comprender las complejidades de este fenómeno, es necesario entender cómo se desarrolla la identidad profesional de los traductores y el rol de la autoeficacia en dicho proceso.

\section{La fenomenología e identidad narrativa}

La fenomenología (Gallagher, 2009; Gallagher y Zahavi, 2012; Zahavi, 2010) concibe la experiencia como un caso único, en donde el individuo interactúa con el mundo tal como se le presenta. En este sentido, la percepción de aquello vivido está determinada por la coherencia entre lo que dicho individuo es y lo que experimenta. En este sentido, la identidad narrativa corresponde al relato coherente del individuo a lo largo del tiempo (Arciero, 2009). Esta identidad se construye mediante una interpretación del grado de continuidad del sí mismo en una experiencia en particular. En otras palabras, la identidad narrativa permite la apropiación activa y constante de vivencias en las que el individuo se reconoce a sí mismo en lo experimentado.
La identidad narrativa presupone la existencia de una historia que permite comprender los diferentes contextos como se nos presentan y, en consecuencia, dar significado a cada momento vivido (Gallagher, 2009; Gallagher y Zahavi, 2012; Zahavi, 2010). Dicho proceso de construcción fenomenológica del significado resulta de la combinación de la subjetividad única e irrepetible del individuo con la que se interpreta lo vivido en carne y hueso, y el lenguaje que utiliza para dar narrar la experiencia (Arciero, 2009; Arciero y Bondolfi, 2009; Ricoeur y Neira, 2013).

En consecuencia,la construcciónidentitaria requiere del conocimiento consciente o inconsciente de lo que la persona vive momento a momento o ipseidad y, a su vez, del conocimiento de la mismidad, es decir, del quién se ha sido, así como de aquellos actos, acciones y emociones que facultan al individuo sentirse el mismo a lo largo de su vida (Arciero 2009; Arciero y Bondolfi, 2009; Ricoeur, 1996). La identidad narrativa tensiona ambos elementos en un ejercicio infinito y cotidiano de hacerse a uno mismo, en donde en el nivel más básico presume la existencia de: 1) narrativas, que organicen la experiencia en una historia; 2) corporalidad, que posibilita el movimiento a propósito de nuestras emociones, y 3) temporalidad, que otorgue una coherencia histórica de los acontecimientos. Estos elementos son los que permiten la interpretación y la documentación de nuestra existencia.

En síntesis, la identidad se construye mediante la apropiación de experiencias en forma de narrativas que presentan una corporalidad situada en constante interacción con el mundo. En este sentido, este estudio aborda el análisis discursivo de las narrativas de los individuos en el momento temporal en que se encuentran: jóvenes que cursan su carrera universitaria.

\section{Identidad en la adolescencia tardía}

Los participantes de este estudio se encuentran en el rango etario de la adolescencia tardía (Papalia et al., 2009). Erikson y Erikson (1997) sugieren 
que, en esta etapa, los individuos tratan de dar sentido a sus acciones pasadas en resultados tangibles presentes. En otras palabras, existe una búsqueda constante por obtener un correlato coherente que demuestre que siguen siendo los mismos. No obstante, la construcción de identidad en dicha etapa se vería insistentemente amenazada por la confusión del rol, marcada por la duda en la ipseidad, es decir, en la interpretación del momento a momento de la vida cotidiana (Erikson y Erikson, 1997). Por otra parte, Marcia (1980) agrega que esta etapa también se caracteriza por una sensación importante de incapacidad para tomar decisiones y relacionarse a sí mismo con una identidad ocupacional.

Lo anterior permite establecer que el estudiante universitario, cuya finalidad es lograr la profesionalización y la especialización, experimentaría dicha confusión en su vida cotidiana, lo que traería consigo un escaso empoderamiento de su rol profesional. No obstante, en la medida en que la persona se capacita y, a su vez, se consolida el pensamiento abstracto, se esperaría que viva el empoderamiento y el reconocimiento afectivo propicio para el posterior posicionamiento como profesional, perfilándose como agente de cambio en su propio devenir histórico y situado.

\section{Definición e interpretación del rol: agente y paciente}

El sentido de agencia es el sentimiento de ser el autor de una acción (Gallagher, 2009), mientras que la posición de paciente de la experiencia es el lugar psicológico en donde se vive desde el padecimiento, sin verse como el responsable de las experiencias. Las narrativas son la forma mediante la cual se determina quién realiza la acción, pues es desde ellas que se abre la posibilidad de hacer propia cada experiencia momento a momento, o de verla en tercera persona, como algo que simplemente sucede o le ocurre a la persona. En este sentido, cómo el sujeto vive cada experiencia adquiere relevancia especial, pues depende de la interpretación de dicha vivencia si el individuo se siente autor-protagonista o personaje de una historia protagonizada por un tercero (Arciero, 2009).
De esta manera, las narrativas cimientan el desarrollo de nuevas formas en que el individuo se siente a sí mismo, por lo que la identidad se vería desafiada por el aquí y ahora, y por el sentido que tiene para el sujeto el construirse como tal (Arciero, 2009; Ricoeur, 1996). Por ejemplo, como se planteó anteriormente, la autoeficacia resultaría de la sensación psicológica sobre la confianza en la ejecución de las competencias y de ser el estudiante quien ejercita y produce los resultados de cara a un objetivo en particular (agente). No obstante, es posible encontrarnos con experiencias en donde no existe la percepción de la autoría en las acciones, viviéndolas desde una posición en donde estas simplemente le suceden al estudiante (paciente).

Bajo esta mirada, el alumno universitario, de cara a su ipseidad, se ve en la dificultad constante de definirse, conocer sus capacidades y limitantes, lo que, a su vez, puede generar un escaso posicionamiento como profesional durante y después del término de su carrera universitaria. No obstante, la capacidad de narrarse a sí mismo posibilita el ejercicio de rehacerse, revelando el sentido de la narración como facilitadora de la identificación y los espacios en que esta se mueve (Arciero, 2009; Arciero y Bondolfi, 2009). Esto permitiría suponer que existiría un compromiso ético en el desarrollo del sí mismo, pues la identidad narrativa puede cambiar el modo de vivirse $y$, a su vez, modificar la disposición hacia una acción determinada, en donde se puede vivir como agente o paciente de las experiencias.

Lo anterior cumple un rol fundamental en la configuración de la identidad. Se espera que, en la adolescencia tardía o adultez temprana, los individuos logren consolidar su identidad, es decir, que exista un compromiso con las elecciones tomadas (Marcia, 1980). Asimismo, dicha etapa vital supone la sensación de autonomía, el autocontrol y la responsabilidad personal, dando cuenta que correspondería a una disposición personal, más que a hechos o acontecimientos aislados (Shanahan et al., 2005). Por lo tanto, la identidad personal, en donde se encontraría la del estudiante universitario con su elección de carrera, supondría 
la capacidad de reconocer las acciones y pasiones, apropiarse, hacerse cargo de cada movimiento y de agenciar la vida del nuevo profesional en formación. Esto posibilitaría un cambio de posicionamiento desde el sujeto paciente que va a la universidad a ser formado, al agente competente y soberano del cual depende una acción comprendida en una historia y es capaz de designarse a sí mismo al significar el mundo.

\section{La dialéctica inward-outward en la interpretación de la experiencia}

Como se ha planteado anteriormente, los individuos están situados en el mundo y, gracias a su corporalidad y temporalidad, son capaces de dar significado a sus experiencias. Un punto crítico en dicho proceso corresponde a la tensión entre la mismidad y la ipseidad de los individuos. Los modos de interpretación emocional se encuentran en un continuo entre corporalidad y la alteridad en que se ancla la experiencia (Arciero y Bondolfi, 2009). En otras palabras, el individuo sitúa sus experiencias hacia las señales de su cuerpo (polaridad inward) o hacia la copercepción del otro (polaridad outward). Los individuos transitan desde un polo a otro según lo que se vive momento a momento, permitiéndoles generar una posición ambivalente entre ambos o doble anclaje, como se representa en la Figura 1 (Arciero y Bondolfi, 2009). Este anclaje experiencial hacia uno mismo o hacia los otros adquiere relevancia, ya que los individuos se reconocen tanto con emociones viscerales, como el miedo, la tristeza, la felicidad, el amor, la rabia, etc., como con otras secundarias, que aparecen de cara a un otro, como la culpa, la vergüenza, la envidia, etc. Dichos reconocimientos emocionales permiten entender quién se es momento a momento,

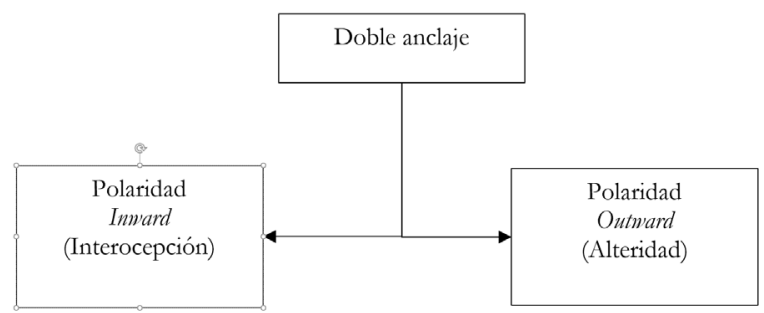

Figura 1 Dialéctica inward-outward si se agencia o no, y cuán eficaz se puede uno sentir en la cotidianeidad.

En síntesis, la identidad narrativa se construye mediante un ejercicio interpretativo de las experiencias en que los individuos dotan de significado a lo vivido. Dicho proceso permite a las personas posicionarse entre las dos polaridades y así encontrar un correlato con quienes han sido y, en consecuencia, poder determinar posibles cursos de acción como, por ejemplo, ejercer su agencia. En el contexto de los estudios superiores, la apropiación de experiencias mediante narraciones se perfila como una instancia de análisis en que los alumnos pueden identificar las experiencias vividas en sus programas de estudio con su profesión y, en consecuencia, agenciar sus acciones en pos de dicha identificación. Este ejercicio de identificación, junto con el sentido activo o pasivo de la agencia, podrían determinar la forma en que una experiencia es interpretada. Por ejemplo, existen alumnos que señalan que las cosas "les pasan", mientras que otros dan un enfoque activo a las tareas y narran "lo que hacen”, lo que finalmente determinaría cómo se desarrolla su autoeficacia en su proceso formativo.

Además, los alumnos de pregrado sistemáticamente se ven desafiados a definirse a sí mismos, reconociendo sus capacidades: lo que pueden hacer y lo que no, así como por lo que pueden responsabilizarse y lo que no. Esto podría llevar a una posición inestable hacia la profesión, durante su formación o incluso posterior a la graduación. Sin embargo, de acuerdo con Arciero (2009), la posibilidad de narrar sus experiencias les permite reconstruir su identidad en su ipseidad. Esto facilitaría la identificación y aportaría a un compromiso ético y profesional a su desarrollo de carrera.

En conclusión, es esperable quelos alumnos universitarios experimenten una confusión en sus vidas cotidianas durante sus estudios y que esto podría gatillar una relativa carencia de empoderamiento hacia sus roles como estudiantes y profesionales. No obstante, a medida que se comprometen con sus elecciones después de tensiones y crisis 
(Marcia, 1980), o sea, experiencias en su formación, su pensamiento abstracto se viviría desde un anclaje emocional determinado para su siguiente posicionamiento como profesionales. En dicho proceso, el conocimiento del ser agente o paciente de su cotidianeidad es crucial en la evolución de su identidad profesional. Más aún, esta etapa también articula sensaciones de autonomía, autocontrol, autoeficacia y responsabilidad personal, que igualmente serían analizadas como proceso, en lugar de productos de eventos o hecho aislados (Shanahan et al., 2005).

\section{Método}

Esta investigación constituye un estudio fenomenológico de carácter cualitativo, desde un paradigma interpretativo, y tiene como propósito examinar las narrativas que los alumnos tienen sobre sí mismos y de sus vivencias en el programa de Traducción, para determinar cómo estas contribuyen a la formación de su identidad profesional y sentido de autoeficacia. Dicha construcción de identidad supone una serie de conceptos cognitivos, actitudinales y biográficos que configuran la subjetividad de la imagen propia traductora y del desempeño asociado a la misma.

\section{Participantes}

Este estudio se llevó a cabo con alumnos de la carrera de Licenciatura en Lingüística Aplicada a la Traducción de la Universidad de Santiago de Chile. Este programa presenta el español como lengua materna (A) desde y hacia la que se traduce al inglés, vale decir, como lengua $\mathrm{B}, \mathrm{y}$ dos menciones adicionales: con portugués, como lengua $\mathrm{B}$ adicional, o japonés como lengua desde la que solo se traduce al español (lengua C).

Los participantes se encontraban cursando el tercer año de su programa de traducción. Es decir, este grupo fue entrevistado en su quinto semestre, antes de iniciar los primeros cursos de traducción, para establecer los constructos asociados a la identidad traductora que han generado hasta este punto previo a la práctica concreta de traducción.
El programa, que consta de cinco años, apunta al desarrollo de las lenguas de trabajo durante los tres primeros años, mientras que los dos últimos presentan los ciclos de especialización en traducción con asignaturas teóricas y prácticas en modalidad directa e inversa. En consecuencia, sus narrativas se desprenden de lo vivido hasta el momento, en el programa, previo a las cátedras de traducción, lo que se plasma en un posicionamiento cognitivo como punto de referencia para estudios posteriores de configuración de identidad de la presente muestra.

Los participantes fueron seleccionados según dos criterios: el primero fue que los participantes debían estar en el rango etario entre 20 y 25 años, mientras que el segundo requisito era que no debían tener deficiencias visuales de cerca, definidas por la Organización Mundial de la Salud (2020) como inferior a N6 o N8 a $40 \mathrm{~cm}$ de distancia. Este último criterio tenía por objetivo evitar que una posible condición visual severa pudiera intervenir significativamente en su interpretación de las experiencias previas y durante el estudio. Lo anterior generó una muestra intencionada, no aleatoria, de 9 participantes, de un posible universo de 32 alumnos; 6 correspondían a la mención de japonés y 3 a la de portugués.

\section{Consideraciones éticas}

Cada uno de los participantes firmó un consentimiento informado de acuerdo con los lineamientos del Comité de Ética institucional de la Universidad de Santiago de Chile, donde se detallaron los objetivos del estudio, así como sus derechos de anonimato y retiro en cualquier momento del estudio.

Se enfatiza que, en el caso de hallar sintomatología psicológica evidente en alguno de los participantes, sea esta causada por la investigación misma o bien por otros factores antecedentes a esta, sin encontrarse en tratamiento previo, se haría la derivación hacia profesionales de atención de salud mental correspondientes. 


\section{Procedimiento}

Los participantes fueron entrevistados en sesiones individuales de entre 35 y 45 minutos cada una. La entrevista, de carácter semiestructurada, contenía dos dimensiones a establecer: el posicionamiento identitario y la formación del traductor profesional. La primera parte consistía en explorar la identidad narrativa de los alumnos, identificando potenciales motivaciones, intereses y significación de su elección de carrera en su cotidianidad. Esto último es crucial, ya que, como señalan Skorikov y Vondracek (2012), la identidad profesional de desarrolla desde la infancia y tiene un punto crucial en la adolescencia, donde los individuos evalúan su vocación, talentos y conocimientos al elegir un camino profesional.

La segunda parte de la entrevista se relaciona con la caracterización del traductor como profesional y el posicionamiento de los participantes en torno a dicho referente, así como un análisis de sus fortalezas y debilidades. La entrevista concluye explorando las aprehensiones y expectativas de los estudiantes ad portas de iniciar su etapa de formación profesional en práctica y teoría de la traducción.

Para rescatar la singularidad de la identidad narrativa, se hizo un análisis de las narrativas de cada uno de los entrevistados, seguido por una caracterización general del grupo, obtenido mediante el método de comparación constante.

Las entrevistas fueron dirigidas por los autores de este trabajo. Estas fueron grabadas y luego transcritas, para su posterior análisis según las dos dimensiones antes descritas. Después, se procedió a la generación de macrocategorías por cada una de las preguntas. Luego, estas fueron estudiadas desde una perspectiva fenomenológica posracionalista, vale decir, centrándose en las experiencias de los participantes y sus representaciones hacia el traductor y su quehacer, así como su conocimiento a priori de la competencia traductora y de la profesión en sí.

\section{Resultados}

El análisis se dividió según las dos dimensiones antes señaladas, a saber, el posicionamiento identitario y la caracterización del traductor. Las contribuciones discursivas de los participantes, presentadas a continuación, son anónimas, y se identifican mediante el uso de números romanos, seguido por el número de intervención dentro de la entrevista.

\section{Posicionamiento identitario}

Esta sección se compone de cuatro partes. Primero se aborda cómo se ven a sí mismos como individuos de manera general. Posteriormente, se indaga cómo conciben su identidad como estudiantes y, luego, más concretamente, el ser estudiante de traducción. Finalmente, la sección concluye con una discusión de su mismidad, vale decir, quiénes han sido y son, y cómo se posicionan en lo vivido en su programa hasta la fecha de la entrevista.

\section{El símismo}

Los participantes viven con dificultad el describirse a sí mismos, partiendo desde la duda hacia una caracterización general poco definida. Se describen como personas tímidas, calmadas, introvertidas y tranquilas. Además, tienden a vivir en solitario y a percibir el desarrollo de la socialización con dificultad.

Eso es un poco ambiguo. No... no sé... soy... [ríe] es que... no sé. Siento que no tengo características particulares; no soy muy buena estudiando, pero tampoco salgo mucho afuera no... no hago cosas... (I 4 ).

Entonces, soy como un poco más, no antisocial, pero no tengo tantas amistades. Entonces, no sé... que igual me cuesta, esto que yo le decía antes que es tratar de ayudar a la gente. Me cuesta, porque no me es fácil acercarme a alguien y hablarle simplemente (vII, 17).

\section{El ser estudiante}

Como estudiantes, se describen como personas motivadas por el estudio de las lenguas y las letras, como también reconocen el valor de su quehacer 
profesional como un elemento fundamental para cruzar barreras idiomáticas.

Bueno, que me emociona, que me alegra mucho tener la oportunidad de poder hacer esto. Que soy una persona que cree, casi hasta el punto de ser demasiado inocente, en el potencial de la humanidad, y me parece que conectar estas culturas es algo muy importante en eso. Entonces, al estar haciendo esto como traductor, siento que estoy haciendo una labor importante para la humanidad como especie. Me llena de esa manera (III, 44).

Lo anterior sugiere que, al enfrentarse a contenidos complejos, los participantes se sienten desafiados por las dificultades que aquello implica. En este sentido, el sobrellevar dichos desafíos actuaría como motivación para la búsqueda de nuevas dificultades, esto es, viven la autoexigencia de modo prerreflexivo, y el miedo, de modo reflexivo. ${ }^{2}$

Igual es una motivación, porque... mientras más... haya cosas que aprender uno puede seguir aprendiendo, entonces... por eso sigo estudiando e investigando, porque si no, nunca voy a... como a lograr eso, es... da miedo, pero... pero es... el que sea un desafío hace que uno quiera superarlo $(\mathrm{v}, 30)$.

No obstante, a pesar de los esfuerzos empeñados, se vive con insatisfacción al respecto, pues se describen como estudiantes esforzados que siempre podrían hacer algo más, reconociendo que el esfuerzo cotidiano en los estudios no es suficiente. Como consecuencia, dicha insuficiencia es vivida con sensaciones descritas como ansiedad, angustia y estrés principalmente.

Bueno, uno siempre queda, o al menos yo siempre quedo con la sensación de que podría dar más estudiando o dedicándole más tiempo. Eso, siento que podría siempre hacer más (vII, 65).

Como... cuando me siento estresada es porque tengo muchas cosas que hacer, muchos deberes y la verdad no me alcanza el tiempo, porque además que soy lenta, no alcanzo a hacer todo y a veces me va mal por eso y me frustro, y ahí es cuando me empiezo a estresar más y más y más y mi rendimiento baja [...] como casi

2 De acuerdo con Arciero y Bondolfi (2009), las experiencias se viven de modo prerreflexivo, vale decir, de manera inconsciente, o reflexiva, esto es, de manera consciente. instantánea. O me empieza a doler el cuerpo cuando estoy estudiando, me duelen los hombros... y, aparte de sentirme mal físicamente, me da como la rabia, la pena y cosas así. Eso siento que igual afecta en mi rendimiento, pero son cosas que a todos los universitarios les pasa [...] no he podido controlar eso porque... la cantidad de cosas me sobrepasan simplemente, y me siento mejor cuando ya va disminuyendo y ahí puedo concentrarme más, rendir mejor (viII, 104, 112, 114).

Es importante destacar que describen un conflicto tanto para descansar como para estudiar: los espacios de descanso y ocio son experimentados desde el deber estar estudiando, mientras que, en el caso de la preparación de trabajos o estudio personal, perciben que el tiempo transcurre y deberían estar haciendo algo al respecto. En ambas situaciones, emerge la culpa como emoción principal, que es vivida desde la sensación de la dificultad de hacerse cargo tanto de sí mismos, en términos de autocuidado, como de los estudios.

Mal porque... si uno sabe que está dentro de sus capacidades, pero no lo hace por motivos que no son tan de peso, entonces, se siente como que es mi culpa y podría ser una mejor estudiante si lo intentara más. Entonces, mal, por eso... no sé si se entiende [...] Sí, pero no es que no... no es que sienta que no hago nada [ríe], solo que, tal vez, podría ser mejor (v, 14 y 16).

La socialización es vivida con miedo a la exposición y emerge desde una vivencia de inseguridad, en donde se sienten interpelados a posicionarse como buenos profesionales.

\section{Sí, me incomoda un poco tener... pero no es porque me incomode ser estudiante de aquí o de la carrera o del idio- ma, sino que me incomoda explicarlo... porque la gente no lo toma en serio, no le ve ninguna... como que no lo piensa más allá, escucha la respuesta y como que quedan igual. No te preguntan nada... no sé, no lo ven como algo serio, como que no saben qué voy a hacer después o qué voy a... qué estoy haciendo ahora, no saben, yo les digo... "estoy estudiando japonés" y es como... "ah, qué bueno" [con desgano y poco interés] $(\mathrm{v}, 56)$.}

\section{Ser estudiante de lingüistica aplicada a la traducción: una identidad colectiva}

Los participantes se sienten situados en una comunidad de identidad propia, diferente a las otras carreras. Describen el programa como colaborativo, 
con pocos alumnos, heterogéneo y que respeta sus propias diferencias. Aparece el sentido de pertenencia, que facilita su sociabilización con otros al percibir a los demás como un semejante, a pesar de las diferencias entre sí. Lo anterior brinda un espacio emocional de tranquilidad, felicidad y comodidad.

Yo diría que me hace feliz, me hace feliz... como que... tengo amigos que son... que están estudiando en carreras que son muy grandes... y me da lata por ellos, saber que no tienen lo que... lo que, como carrera, nosotros tenemos $[. .$.$] me sentía sola a ratos, como que me fal-$ taba como... como identificarme dentro de un grupo. Y yo creo que eso es lo que obtuve cuando entré a la carrera. Cosa que no la esperaba, en realidad, no era como una de mis metas... en el minuto en el que decidí inscribirme. Me sentía como plena, pero... como que sentía que faltaba algo... que faltaba como este elemento de unidad de grupo (II, 60 y 66).

Asimismo, se describen como reflexivos, de aprendizaje rápido y de interés por lo nuevo. No obstante, los alumnos se ven como extraños para los demás, como "un espécimen", pues deben explicar de qué trata la carrera y se ven interpelados a hablar bien de lo que están estudiando. Se sienten evaluados y desde ahí emergen dos vertientes: la primera trata de la tristeza y duda por la devaluación de las demás personas hacia la carrera. La segunda alude a la felicidad y el goce debido a la identificación entre ellos y con lo que se estudia, lo que les genera un sentimiento de orgullo de "ser los embajadores" y llevar una bandera de visibilidad para los otros.

Bueno, yo creo que cada uno... obviamente, todos somos distintos, pero también cumplimos como con factores que son bien similares, que también tienen que ver con cómo soy yo... que, de forma similar, también somos muy... como tranquilos, como que tendemos a reflexionar, más que a... como a pensar... o hablar de inmediato. Y eso. [...] Yo diría que somos reflexivos... que aprendemos rápido y que disfrutamos aprender cosas nuevas. [...] Yo diría que me hace feliz, me hace feliz... como que... tengo amigos que son... que están estudiando en carreras que son muy grandes... y me da lata por ellos, saber que no tienen lo que... lo que, como carrera, nosotros tenemos [...]. Entonces, me siento como una representante de la carrera, así como... "ya, tengo que dejar bien a la carrera o... tengo que hacerla parecer interesante y todo eso" (II; 13, 58, 60 y 70).

\section{Mismidad: cómo han vivido y viven su programa}

Los participantes se describen a sí mismos desde un proceso de transformación que se inicia con las motivaciones al momento de entrar a estudiar Lingüística Aplicada a la Traducción. Además, durante el transcurso de los semestres de estudio, destacan el aprendizaje y el reconocimiento de las dificultades. En otras palabras, explicitan lo que pueden agenciar y lo que no, de cara a un futuro incierto e inexcusable.

Las narrativas sobre el posicionamiento de los participantes se relacionan con su mismidad y cómo esta se vincula con sus motivaciones, objetivos y creencias pasadas, actuales y futuras.

1. Pasado. Entre las motivaciones para estudiar, aparecen dos aristas principales que facilitan describir por qué les interesó y por qué se han mantenido cursando el programa de estudio: la facilidad para aprender los idiomas y el desafío de estudiar lenguas que son complejas. La primera aparece como un indicador de competencias lingüísticas previas, en donde reconocen siempre haber tenido capacidad para aprender idiomas, ya sea viendo películas, caricaturas o leyendo cómics japoneses. La segunda, como se señaló anteriormente, implica ver la carrera como un área difícil de estudiar, lo que los motivaría a cumplir con el desafío de superar dificultades, apareciendo así la autoexigencia prerreflexiva como recurso fundamental.

La principal motivación [es] superarme, estudiar algo y tener una carrera. Pero el motivo de por qué elegí esto, porque siempre, toda mi vida, me han gustado los idiomas. Cuando chica me gustaba el inglés y cuando entré a esta carrera, me empezó y terminó de gustar portugués. $\mathrm{O}$ sea, no terminó, me empezó a gustar portugués (VIII, 8).

2. Presente. En la actualidad, aparece la intención de rendir adecuadamente en sus estudios. Sin embargo, en ocasiones se sienten frustrados y angustiados por la falta de tiempo. Además, se problematiza el hacerse cargo de aquellos aspectos 
personales, como la timidez, la introversión y el miedo a la exposición, reconociéndolos como aspectos fundamentales que deben agenciar para afrontar el futuro. Adicionalmente, se sienten expectantes y con miedo a equivocarse, tanto por la elección del idioma (japonés o portugués) como en las mismas traducciones y el ingreso al mundo laboral.

\begin{abstract}
Mi problema creo que es que no se me da tan bien relacionarme con las personas, o al menos iniciar esa relación. Me cuesta mucho, entonces, en este trabajo que puede ser tan aislado o dependiendo de cómo se haga, creo que sería un problema eso de no tener contactos o no tener tanta gente a la que recurrir o gente que recurramos. Una cosa así (VII, 63).

Eso me asusta, que tener que, en general... relacionarme con otras personas... y el hecho de tener que... es que no es porque sea esto específicamente, sino que el mundo laboral, en general, parece un... parece aterrador [ríe], porque uno tiene que valerse por sí mismo y eso... no sé si soy tan capaz de hacerlo. O sea, supongo que sí, pero no me gusta $(\mathrm{V}, 90)$.
\end{abstract}

3. Futuro. A pesar de referirse a la práctica y al ingreso al mundo laboral como desconocido, se proyectan como profesionales íntegros, rigurosos, minuciosos, éticos y que han mejorado su desempeño en relación a cómo se ven ahora.

\begin{abstract}
Me gustaría ser un profesional íntegro, o sea, estar bien segura de mis capacidades para traducir. También, por ejemplo, no salir de la carrera y tener dudas con la lengua, o si las tengo, si es que las llego a tener, que yo creo que sí puede pasar (a todos les pasa), es ser capaz de buscarlo y salir de la duda. También me gustaría, no solo en el aspecto como de conocimiento de materia, también me gustaría tener más personalidad para poder aplicarlo a un futuro trabajo quizá [...] por ejemplo, tratar con personas. Por ejemplo, una intérprete, hay que igual interactuar con personas [...] para la práctica que voy a hacer, voy a necesitar tener mucha personalidad, desenvolverme bien con las personas y eso me gustaría terminar haciendo bien, también (VIII, 42, 44, 46).
\end{abstract}

En conclusión, en esta dimensión los alumnos de traducción presentan características personales retraídas y tímidas, que se ven amparadas en un sentido de colectividad del programa, que a su vez se enuncia como instancia de desarrollo personal, pero también como agente que tensiona la identidad de los alumnos ante los no miembros de la comunidad. En este sentido, los estudiantes hacen énfasis en el rol que tienen como representantes del grupo con el que se identifican. Hay una coherencia temporal entre las razones de ingreso al programa y las tensiones con un futuro que, aunque incierto, se perfila como oportunidad de consolidación de la idealización personal que tienen de sí mismos y el rol del traductor.

\section{Caracterización del traductor profesional}

Esta segunda parte examina la caracterización del traductor profesional, la valoración que los estudiantes otorgan a la formación recibida hasta el momento de la entrevista y sus expectativas ante la especialización en traducción de los próximos años.

\section{Caracterización del traductor profesional}

En relación con el primer punto, las narrativas de los alumnos perfilan al traductor como un profesional seguro de la labor que realiza. Esta seguridad se logra mediante la adquisición de un conocimiento teórico sólido sobre traducción, así como por el manejo de diversas temáticas. Del mismo modo, se enfatiza el manejo de las lenguas B y C, para poder generar traducciones precisas.

Por otra parte, verbalizan algunas habilidades profesionales y personales relevantes para el desarrollo de su profesión, en particular la capacidad de generar redes de contacto y apoyo para la realización de trabajos de traducción, así como la capacidad de trabajo colaborativo.

Se conceptualiza la traducción como un acto colectivo y no individual. Adicionalmente, se concibe la tarea traductora como una actividad que requiere de un desarrollo profesional constante, potenciada por un deseo propio de perfeccionamiento.

Respecto a las características personales, se destaca la integridad, comprendida como una combinación de varios atributos, a saber, la flexibilidad, 
el compromiso, la sistematicidad y la capacidad reflexiva.

Me gustaría ser un profesional integro, o sea, estar bien segura de mis capacidades para traducir. También, por ejemplo, no salir de la carrera y tener dudas con la lengua... también me gustaría tener más personalidad para poder aplicarlo a un futuro trabajo quizá. Que siempre esté aprendiendo, que esté siempre atento a los detalles. Que ojalá... también que tenga como comunicación con la comunidad de traductores para poder como ayudarnos entre sí. Como una persona bien versátil (VII, 21, 28).

[El traductor es alguien] flexible con su trabajo, con lo que está haciendo, y alguien que pueda colaborar con los demás también, si fuera necesario. Me gustaría ser, buena. O sea, tener una base bien firme y poder hacer las cosas bien... que [las] traducciones estén correctas, lo más correctas posible... como aspirar a la excelencia... que sea de calidad el trabajo (v, 28, 31).

En cuanto a los factores privados que intervienen en la formación profesional de un traductor, los estudiantes señalan inicialmente que la capacidad de generar redes de contactos es fundamental para establecer potenciales oportunidades laborales en el futuro. Esto coincide con la noción de que la traducción es un acto individual inserto en una colectividad. Adicionalmente, apuntan al esfuerzo y la perseverancia como ejes sostenedores de la motivación en el aprendizaje y el dominio de las lenguas de trabajo. En relación con esto último, se enfatiza una metodología de trabajo acuciosa y sistemática, lo que demanda una autogestión del tiempo que el estudiante debe asumir.

Lo anterior se asocia con la capacidad de responsabilizarse para suplir carencias y debilidades en el proceso de enseñanza-aprendizaje. $\mathrm{Al}$ respecto, en la búsqueda de oportunidades de práctica significativa, la proactividad figura como una habilidad crucial a desarrollar.

Por último, destacan el rol que tiene el conocimiento en diversas áreas de estudio para lograr realizar traducciones de manera eficiente.

[...] cuando empecé la carrera, yo tenía la impresión de que... habían ciertos profes que no se desempeñaban tan bien como otros profes y que eso iba a impedir que yo aprendiera mejor; pero esto ya fue en primer año; ahora en tercero, sigo pensando que hay profes que no son tan competentes como otros profes, pero que aun así, yo igual puedo avanzar como por mí misma, que no es necesario que me ligue tanto al programa de un profesor o a lo que un profesor estima conveniente que aprendamos (II, 48).

[...] en lengua portuguesa, no tener las suficientes instancias para practicar la lengua, por ejemplo, o no sentirme con suficiente contenido. $\mathrm{O}$ a veces siento que están repitiendo todo, entonces, como un repaso de los otros años. Entonces, a veces siento que eso me puede afectar, como sentirme débil en el aspecto de la lengua (viII, 36).

Por otra parte, los factores externos se agrupan en tres categorías: metodológicos, curriculares y personales. Los primeros están relacionados con el programa de estudio. Al respecto, señalan que existen problemas didácticos en las clases de las lenguas B y C. En concreto, se cuestiona la idoneidad de los profesores para impartir dichas cátedras, en particular en el caso de las asignaturas relacionadas con el área de japonés. La dificultad que presenta este idioma se debe a la falta de instancias de práctica significativa con hablantes nativos.

Adicionalmente, señalan que el grupo tiene un papel relevante en el proceso de aprendizaje.

Por último, se acusa que las convenciones propias de las asignaturas de lingüística constituyen un desafío cognitivo importante para algunos estudiantes.

Hay algunos profesores que tal vez no... siento que no son los adecuados para enseñarnos ciertas cosas y... eso va a ser bastante difícil como, en cuanto a la motivación o aprender ciertas cosas, y que son habilidades importantes para el traductor... Sobre todo en el área de japonés, siento que hay profesores que tal vez no son muy buenos... sobre todo con los profesores de lengua... hay algunos que tal vez no son profesores como por carrera y no tienen una metodología para enseñar, $\mathrm{u}$ otros profesores que tal vez no hablan tan bien japonés y que eso hace bastante difícil poder aprender el idioma y, realmente, aprender el idioma es importante para un traductor $(\mathrm{I}, 74,75,77)$. 
En cuanto al aspecto curricular, los alumnos señalan que el número de idiomas a aprender no permite que se pueda destinar suficiente tiempo a cada uno para aprenderlos de manera óptima. Además, se enfatiza en el alto nivel de exigencia que posee el programa en cuanto al rendimiento.

[...] la cantidad de ramos [cursos]. Sí, yo creo que el hecho de algunos semestres tener tantos ramos, hace que no pueda enfocarme apropiadamente o enfocarme en lo que yo querría enfocarme más a fondo (III, 50).

[...] yo siento que la exigencia está bien, pero a veces como que el trabajo sobrepasa lo que uno puede hacer (vi, 38).

Por último, en el ámbito personal, los alumnos nombran los impedimentos fisiológicos (accidentes que provoquen ceguera o discapacidad), problemas interpersonales (discusiones o fallecimiento de familiares) y compromisos extracurriculares (trabajo, voluntariado o cuidar a algún familiar) como posibles factores que atentan contra el desarrollo profesional. Del mismo modo, se plantea que el deseo de un egreso oportuno del programa pueda verse amenazado por posibles paralizaciones (paros) estudiantiles en la Universidad.

\section{Valoración de la formación a la fecha}

En sus narrativas, los estudiantes se muestran tensionados en cuanto a su satisfacción con la calidad de la preparación ad portas del inicio de la formación en traducción. Algunos de los participantes destacan la estructuración de la malla curricular y vislumbran potencial en las posibles alternativas profesionales futuras. Otros participantes señalan no sentirse preparados en las lenguas B y C. Lo anterior obedece a problemas metodológicos y didácticos experimentados en los cinco niveles del programa. $\mathrm{Al}$ respecto, acusan repetición de contenidos y textos de estudios desactualizados.

Se aprecia una distinción entre ciertos profesores que aparentemente cumplen con las expectativas de los estudiantes, mientras que otros son seriamente cuestionados en su actuar docente, en particular en su capacidad de generar instancias de práctica significativa. En este sentido, hay una valoración de la producción propia, concebida como la aplicación de los conocimientos desarrollados en la configuración de una identidad propia en las lenguas B y C.

\section{[...] de los ramos de japonés también siento un poco de frustración de... que siento que de repente la didáctica no se adapta muy bien a enseñar bien la lengua, como nos apegamos mucho al Minna no Nihongo [texto de en- señanza de japonés], que está muy desactualizado, que cuesta mucho aprender japonés con eso, que al final termina siendo más estudio de uno que lo que entrega la universidad (IX, 52).}

[... y nos frustraba mucho que el profesor utilizara el... que él dijera: "ya, utilicemos esta lección del Minna no Nihongo",y la leyeray dijera: "ya, vayan viendo dónde van los acentos”; nosotros los íbamos poniendo y él decía una palabra y él decía: "no, yo creo que el acento va acá y que va acá", y a veces los iba cambiando y como que nosotros no le encontrábamos la ciencia, porque era como literalmente tratar de adivinar dónde estaba para nosotros, porque después aparecía la prueba y nos aparecía un montón de palabras y nosotros estábamos como "pucha, esto lo escuchamos una vez, ¿cómo voy a saber dónde está el acento acá si no nos enseñaron como ninguna técnica para aprenderlo?” (vII, 38).

[...] aprendo mucho produciendo más que repitiendo cosas, y la verdad es que repetimos hartas formas en las clases y es poco lo que producimos. Siento que, en ese sentido, me gustaría que hubiera más y en todo aspecto, porque recuerdo que el semestre antes anterior, el primer semestre del año pasado, tuvimos dos ramos que eran los de cultura, que nos hacían hacer ensayos y hartas cosas escritas, producidas por uno, y... ahí sentía que en verdad estaba como, no sé, aplicando todo lo que había aprendido... sé que me iría terrible en japonés, pero me gustaría intentarlo $[\ldots](\mathrm{v}, 40)$.

Adicionalmente, se observa una diferencia en las conductas de entrada de los alumnos al ingreso a la carrera. Mientras que algunos alumnos critican la estructura y la planificación de las asignaturas de expresión oral y escrita en español, otros la valoran, en cuanto a que les han posibilitado desarrollar habilidades sociales que no hubieran podido consolidar de otra manera.

[...] el ramo de expresión oral y escrita, siento que pudo haber sido bastante provechoso para aprender 
formalidades al escribir, pero se enfocó muy poco en esas cosas como ortografía y puntuación, y luego fue a otros temas como que no eran muy útiles (IX, 53).

Con ramos como "expresión oral", me ayudó harto a poder vencer ese terror a hablar en público. En eso específicamente me ayudó más (VIII, 50).

Por último, los alumnos concuerdan en el alto valor de las asignaturas de cultura en el programa, pero señalan limitaciones en cuanto a: (1) tiempo para la entrega del contenido y (2) preparación de los profesores que imparten la asignatura.

\section{Expectativas sobre el ciclo profesional}

Los alumnos cuestionan la articulación curricular del programa, en particular el que las asignaturas de traducción se integren a partir del sexto nivel en adelante. En este sentido, expresan que este cambio requiere una transición más gradual. A pesar de esto, se evidencia un claro interés de la mayoría de los participantes en iniciar el ciclo de traducción y relacionarse con estrategias que les faculte para enfrentar diferentes tipos textuales de manera precisa. Esto, según algunos, les posibilitaría desarrollar cierta seguridad para poder enfrentar los textos a traducir de mejor manera, así como aprender sobre diversas áreas temáticas.

Por otro lado, existe inseguridad en cuanto a las capacidades propias para afrontar las asignaturas de traducción de manera adecuada durante los cursos. No obstante, los participantes perfilan la práctica como instancia para consolidar sus habilidades y así poder concluir sus estudios en los plazos establecidos por el programa.

[...] me ponen nerviosa, porque siento que quizás mis traducciones no sean las mejores. Entonces, me da nervios no ser tan eficiente como debería como traductora ahora al empezar a darme cuenta de que quizás las cosas no me están saliendo bien (vII, 46).

[...] me siento emocionada de como que por fin poder ver esto de la traducción en sí. Espero que, espero entender toda la parte como teórica también y tener mucho en práctica. Espero que sea como equilibrado esos dos aspectos, que sean equilibrados, también, para yo tam- bién poder desarrollar mis capacidades y también como mejorar en lo que siento que no estoy muy bien (VI, 46).

Resulta interesante que, en algunos casos, se evidencia, en este nivel de aprendizaje, conciencia de la ética del traductor y la voz del mismo en la traducción.

[...] como por ejemplo cuando... si hago una traducción, tener en cuenta como a quién va dirigida la traducción, quién es el autor, qué es lo que quiere transmitir y reflejar como su punto de vista y no como meterme en eso si es que no tengo como la autoridad de hacerlo, que es como la parte ética de la traducción; y también que, si eso pasa, que no pase porque podría ser un error, así que quiero poder saber identificar esos errores para que no pase esa situación (vI, 39).

\section{Discusión}

Las narraciones analizadas sugieren que los participantes tienen una vivencia de sí mismos, en donde se recalca constantemente el miedo a equivocarse, la exposición, la culpa y la vergüenza. De acuerdo con su narrativa, se reconocen a sí mismos desde emociones secundarias y algunas primarias, lo que daría cuenta de un doble anclaje psicológico. En concreto, los alumnos transitarían hacia la polaridad outward, es decir, todas aquellas emociones que emergen cuando existe la presencia de otro, real o simbólico. En este sentido, es posible visualizar que los participantes tienen una vivencia de sí mismos, en donde se recalca constantemente el miedo a la exposición a un posible error, la culpa y la vergüenza.

Desde este punto de vista, los estudiantes van construyendo una identidad, en donde son particularmente sensibles a los ojos de los demás, sintiéndose interpelados a ser responsables, éticos, rigurosos, etc. El que se reconozcan de esta forma pareciera que tensiona sus deseos, anhelos y posibilidades, pues al estar doblemente anclados, terminan generando una brecha entre el qué quieren de sí mismos de cara al quién deben ser. En consecuencia, el sentido de agencia y autoeficacia se encuentra constantemente apelando a un otro y al miedo como puntos de referencia, desconociendo en ocasiones que tanto la experiencia como el quehacer académico les pertenece. 
Asimismo, dicho doble anclaje facilitaría la permanencia en un estado de entender que quieren ser profesionales íntegros, pero que, desde el cómo, se sienten visceralmente atemorizados y paralizados, lo que les dificulta poder agenciar al menos en la actualidad.

Por otra parte, el desarrollo de una identidad grupal entre los pares los faculta para que se sientan seguros, cómodos y con un sentido de pertenencia, que diferiría al de otras carreras universitarias. Este sentido de pertenencia les permitiría vivirse con adecuación afectiva, o sea, que lo que sienten dentro del grupo es pertinente, viviéndose a sí mismos como sujetos situados en un contexto que posibilita anclar las emociones libremente. No obstante, tal identidad pareciera vivirse con dificultad al enfrentar a personas externas, pues sienten que tienen que explicar y exponer constantemente la validez e importancia de lo que estudian. Pareciera que esta dificultad marca un punto significativo en cuanto a sentirse visibles y validados de cara a los demás, pues es una de las experiencias que viven con inseguridad.

Todos los participantes reconocen tener dificultad para socializar y se caracterizan por ser personas tímidas, calladas o de pocas palabras. Es aquí en donde emerge un conflicto, pues aquello es vivido desde el padecimiento, es decir, desde un lugar psicológico, en donde dichas características personales serían difíciles de superar y formarían parte de una cualidad perenne a lo largo del tiempo, que coarta su libertad psicológica. Asimismo, podría señalarse que la tranquilidad que existe al sentirse pertenecientes y validados en un grupo de personas, evidencia el no verse exigidos a hacerse cargo de su socialización.

La inseguridad puede ser interpretada principalmente desde el reconocimiento afectivo hacia la alteridad: los demás son vistos como personas que devalúan la elección de su carrera. Al ser particularmente sensibles a esta mirada y, a su vez, esta, al ser constantemente cambiante, se pierden a sí mismos como punto de referencia, corriendo el peligro de que los demás comiencen a serlo. Desde ese lugar es donde se ven exigidos a explicar de qué se trata y lo importante que es estudiar Lingüística Aplicada a la Traducción. Un ejemplo de aquello es la dificultad que hay para describirse a sí mismos en comparación a cómo se reconocen como estudiantes, en donde es posible levantar más narrativas y significados asociados.

Como se ha señalado en la literatura (Erikson y Erikson, 1997; Marcia, 1980), es posible evidenciar las diferentes tensiones emocionales que emergen a propósito del tránsito entre el compromiso ético emocional y, a su vez, lo que se espera de un estudiante exitoso. Aparentemente, dicha tensión se vive entre la alteridad como referente de la experiencia y el enmudecimiento de emociones viscerales, como la felicidad, la rabia, la tristeza, entre otras. No obstante, la tensión mencionada pareciera resolverse al encontrarse con otros semejantes con quienes se vinculan a propósito de sus similitudes identitarias: timidez, introversión, dificultad en la socialización, miedos, etc.

Si bien la identidad colectiva resulta un factor protector y sanador de cara a la brecha emocional, es este un estado psicológico en el cual aparentemente se sienten atrapados, reconociendo que se debe socializar para posicionarse como profesionales. En este sentido, la identidad colectiva actúa como una experiencia universitaria más amena en comparación con la que, de acuerdo con su relato, padecen (miedo a la exposición, timidez, introversión, etc.). En consecuencia, no existirían acciones que posibiliten agenciar esta dificultad, para hacerse cargo del presente y del futuro como estudiantes y profesionales.

El sentido de agencia del actuar de los estudiantes también estaría enmudecido, pues pareciera que efectivamente pueden estudiar y obtener buenas calificaciones, que es lo que sienten que deben hacer. No obstante, hay una autoexigencia prerreflexiva que no dejaría reconocer aquello, lo que daría como resultado la vivencia de que las notas y el rendimiento no serían suficientes. La escasez de experiencia de sentimiento de agencia permitiría comprender el hecho de vivirse a sí mismos como 
estudiantes que no son eficaces a la hora de cumplir con su rol académico, social y profesional.

Lo anterior se condice con la idealización de la figura del traductor, en donde se le posiciona como un individuo íntegro, con una gran cantidad de virtudes que ellos parecen no tener. En particular, la explicitación de la necesidad de redes de contacto y la capacidad de trabajo colectivo tensiona la comodidad que sienten al ser parte de la carrera, con su futuro profesional, que se ve mucho más incierto. Otro ejemplo de esta tensión se evidencia en la identificación y la categorización de problemáticas curriculares y metodológicas del programa, en donde depositan la mayor cantidad de problemas que les impedirían convertirse en traductores. Si bien esto supone una serie de consideraciones desde la estructura y la metodología de los cursos, las narrativas sugieren dos posicionamientos: por una parte, algunos alumnos culpan a la alteridad desde una pasividad en la acción, es decir, no se encuentran dispuestos a agenciar para enfrentar estos desafíos en su ipseidad; por otra, algunos alumnos utilizan estas dificultades contextuales presentes en la alteridad como insumo predicativo que permite impulsar la agencia para tomar acciones que favorezcan sopesar dichos impedimentos o carencias metodológicas.

\section{Conclusiones}

Este trabajo corresponde a un primer acercamiento al posicionamiento de un grupo de alumnos de Traducción que inician su proceso formativo en la traducción en inglés como lengua B. Las narrativas de los estudiantes sugieren que existe una baja agencia prerreflexiva o, en algunos casos, esta está ausente. Además, se evidencia un doble anclaje emocional, pues admiten que en ocasiones no agencian tanto como esperarían hacerlo, ya sea por miedo, deseo propio o por causas enmarcadas en la alteridad, por ejemplo, estructura curricular del programa, metodología y didáctica de las asignaturas, y maximización del uso de su tiempo de trabajo autónomo. Esto último perfila la autoeficacia a este nivel como una actitud positiva de agencia hacia el programa de estudios, así como un buen desempeño en las lenguas estudiadas.

Por otra parte, prima una identidad colectiva como un factor que ofrece protección ante la confrontación con los otros no miembros del programa de estudios. No obstante, dicha protección mancomunada no es efectiva frente a la autoexigencia prerreflexiva de algunos alumnos. Dicha autoexigencia no les permite valorar sus buenos resultados académicos como parte de su desarrollo profesional, ya que siguen pensando que no poseen las competencias necesarias y que cualquier esfuerzo no es suficiente.

En consecuencia, se podría perfilar al alumno de tercer año del programa como un individuo reservado, con dificultades para socializar y agenciar su desarrollo personal individual y profesional en el programa. No obstante, la colectividad y el sentido de grupo permiten que pueda sobrellevar ciertas situaciones y generar un posicionamiento más activo hacia la alteridad.

Este tipo de insumo resulta de mucha utilidad para la planificación didáctica de las unidades de traducción, pues al conocer la identidad del alumno que vive el programa, se podría llegar a una dinámica que potencie un reconocimiento y una agencia de emociones viscerales y secundarias, que posibiliten que los alumnos se hagan responsables de su proceso de aprendizaje, como señalan en su narrativa.

En ese sentido, se hacen necesarios dos reflexiones desde el currículum: (1) que el diseño de asignaturas dentro del programa permita una transición libre desde una polaridad outward a inward para potenciar la agencia de los alumnos, y (2) planificar las asignaturas desde una mirada constructivista, donde la negociación de significados se realice de forma colectiva, de manera que se potencien el desarrollo de competencias profesionales y habilidades sociales. Esto último sería de especial relevancia para poder generar las redes de contactos que los alumnos esperan de un traductor profesional. 


\section{Referencias}

Arciero, G. (2009). Tras las huellas de sí mismo. Amorrortu.

Arciero, G., y Bondolfi, G. (2009). Selfhood, identity and personality styles. Wiley Blackwell.

Atkinson, D. P. (2012). Freelance translator success and psychological skill: A study of translator competence with perspectives from work psychology (Tesis doctoral). University of Auckland, Nueva Zelanda.

Atkinson, D. P. (2014). Developing psychological skill for the global language industry: An exploration of approaches to translator and interpreter training. Translation Spaces, 3, 1-24. https://doi.org/10.1075/ ts.3.01atk

Diéguez, M. I., Lazo, R. M., y Quezada, C. (2014). Estudio de mercado de la traducción en Argentina, Chile y España: perfil académico y profesional de los traductores. Onomázein, (30), 70-89. https://doi.org/10.7764/ onomazein.30.6

Diéguez, M. I., Lazo, R. M., y Quezada, C. (2015). Perfil del teletraductor en Argentina, Chile y España: las TIC aplicadas a la práctica profesional. Onomázein, (31), 1-19. https://doi.org/10.7764/onomazein.31.2

Erikson, E. H., y Erikson, J. M. (1997). The life cycle completed. W.W. Norton.

Gallagher, S. (2009). Philosophical antecedents of situated cognition. En P. Robbins y M. Aydede (Eds.), Cambridge handbook of situated cognition (pp. 35-51). Cambridge University Press.

Gallagher, S., y Zahavi, D. (2012) The phenomenologicalmind. Routledge.

Haro-Soler, M. M. (2017a). ¿Cómo desarrollar la autoeficacia del estudiantado? Presentación y evaluación de una experiencia formativa en el aula de traducción. Revista Digital de Investigación en Docencia Universitaria, 11(2), 50-74. http://dx.doi.org/10.19083/ ridu. 11.567

Haro-Soler, M. M. (2017b). Teaching practices and translation students' self-efficacy: A qualitative study of teachers' perceptions. Current Trends in Translation Teaching and Learning E, 4, 198-228.

Haro-Soler, M. M. (2018). Las creencias de autoeficacia del estudiantado de Traducción: una radiografía de su desarrollo (Tesis doctoral). Universidad de Granada.
Hubscher-Davidson, S. (2018). Translation and emotion. A psychological perspective. Routledge.

Marcia, J. E. (1980). Identity in adolescence. En J. Adelson (Ed.), Handbook of adolescent phychology (pp. 159187). Wiley.

Muñoz, R. (2014). Situating translation expertise: A review with a sketch of a construct. En J. W. Schwieter y A. Ferreira (Eds.), The development of translation competence: Theories and methodologies from psycholinguistics and cognitive science (pp. 2-57). Cambridge Scholars Publishing.

Organización Mundial de la Salud (2020). Cegera y discapacidad visual. https://www.who.int/es/news-room/ fact-sheets/detail/blindness-and-visual-impairment

PACTE (Proceso de Adquisición de la Competencia Traductora y Evaluación). (2017). PACTE Translation Competence Model. En A. Hurtado Albir (Ed.), Researching translation compentece by PACTE Group (pp. 35-41). John Benjamins.

Papalia, D. E., Olds, S. W., Duskin, F. R., Olivares, S. M., Padilla, S. G. E. (2009). Psicología del desarrollo: de la infancia a la adolescencia. McGraw-Hill.

Ricoeur, P. (1996). El sí mismo como otro. Siglo Veintiuno Editores.

Ricoeur, P., y Neira, C.A. (2013). Sí mismo como otro. Siglo Veintiuno.

Shanahan, M., Porfeli, E., y Mortimer, J. (2005). Subjective age identity and the transition to adulthood: When do adolescents become adults? En R. A. Settersten y R.G. Rumbaut (Eds.), On the frontier of adulthood: Theory, research, and public policy (pp. 225-255). University of Chicago Press.

Skorikov, V. B., y Vondracek, F. W. (2012). Occupational identity. En S. J. Schwartz, K. Luyckx y V. Vignoles (Eds.), Handbook of idenitty theory and research (pp. 693-714). Springer.

Subsecretaría de Relaciones Económicas Internacionales. (2020). Chile y el comercio de servicios. https://www. subrei.gob.cl/servicios/

Tan, C. P., Van der Molen, H. T. y Schmidt, H. G. (2016). To what extent does problem-based learning contribute to students' professional identity development? Teaching and Teacher Education, 54, 54-64. https:// doi.org/10.1016/j.tate.2015.11.009 
Way, C. (2009). Bringing professional practices into translation classrooms. En I. Kemble (Ed.), The changing face of translation (pp. 131-142). University of Portsmouth.

Wood, R. E., Atkins, P., y Tabernero, C. (2000). Self-efficacy and strategy on complex tasks. Applied Psychology: An
International Review, 49(3), 430-446. https://doi. org/10.1111/1464-0597.00024

Zahavi, D. (2010). Naturalized phenomenology. En D. Schmicking y S. Gallagher (Eds.), The handbook of phenomenology and cognitive science (pp. 2-19). Springer.

Cómo citar este artículo: Singer, Néstor, López, Vania, y Basaure, Rosa. (2020). Identidad profesional en estudiantes de traducción chilenos: posicionamiento, percepciones y valoraciones iniciales. Íkala, Revista de Lenguaje y Cultura, 25(2), 455-473. https://doi.org/10.17533/udea.ikala.v25n02a01 